\title{
Aspirin enhances opsonophagocytosis and is associated to a lower risk for Klebsiella pneumoniae invasive syndrome
}

\author{
Chen-Hsiang Lee ${ }^{1,2}$, Lin-Hui Su, ${ }^{3,4}$, Jien-Wei Liu ${ }^{1,2}$, Chia-Chi Chang ${ }^{1}$, Rong-Fu Chen ${ }^{5}$ and Kuender-D Yang ${ }^{*}$
}

\begin{abstract}
Background: Klebsiella pneumoniae (KP) expressing hypermucoviscosity phenotype (HV-KP) has abundant capsular polysaccharide (CPS) and is capable of causing invasive syndrome. Sodium salicylate (SAL) reduces the production of CPS. The study was aimed to investigate the relationship between aspirin usage and KP-mediated invasive syndrome and the effect of SAL on HV-KP.

Methods: Patients with community-acquired KP bacteraemia were prospectively enrolled. KP-M1, a serotype-K1 HV-KP clinical isolate, was used in the following experiments: CPS production, HV-KP phenotype, and the effect of SAL on neutrophils phagocytosis. The effect of oral aspirin intake on the leukocyte bactericidal activity was evaluated.

Results: Patients infected by HV-KP and diabetic patients with poor glycemic control were at an increased risk for invasive syndrome $(p<0.01)$; those who had recent use of aspirin $(p=0.02)$ were at a lower risk. CPS production was significantly reduced in the presence of SAL. The HV-KP phenotype and resistance to neutrophil phagocytosis were both significantly reduced in the KP-M1 after incubation with SAL $(p<0.01)$. Aspirin treatment significantly enhanced the killing of KP-M1 by leukocytes $(p<0.01)$.
\end{abstract}

Conclusion: Treatment with SAL significantly reduces CPS production in HV-KP, thereby contributing to leukocyte phagocytosis and bactericidal activity against this pathogen.

Keywords: Salicylate, K. pneumoniae, Hypermucoviscosity, Phagocytosis, Invasive syndrome

\section{Background}

Primary Klebsiella pneumoniae (KP) liver abscess occurs most often in patients with type 2 diabetic mellitus $(\mathrm{DM})$, and can present with the following metastatic complications: bacteraemia, meningitis, endophthalmitis, and necrotising fasciitis [1,2]. Glistening mucoid colonies with a viscid consistency are usually formed by KP that are cultured on agar media [1-3]. Hypermucoviscous KP (HV-KP) causes a unique constellation of symptoms, and is associated with the development of invasive syndrome $[2,3]$. The HV-KP strain is resistant to phagocytosis by neutrophils, an important characteristic that may contribute to the dissemination of infection [4-6]. The capsular polysaccharide (CPS) contributes to the mucoid phenotype, and

\footnotetext{
* Correspondence: yangkd.yeh@hotmail.com

${ }^{5}$ Department of Medical Research, Show Chwan Memorial Hospital, 6 Lukong Road, Lugang, Changhua 505, Taiwan

Full list of author information is available at the end of the article
}

has been identified as a determinant of KP infection, suggesting that CPS plays a role in the development of invasive syndrome $[7,8]$. CPS also has been used to develop a serotyping system for KP isolates, and currently 77 capsular serotypes have been identified. Compared to those belonged to non- $K_{1}$ serotypes, isolates of the serotype $K_{1}$ were significantly more virulent and more likely to be associated with KP-mediated invasive syndrome [2,7].

Chemotherapeutic agents that reduce CPS production may be effective as an adjunct therapy for HV-KP infection. Therapeutically safe concentrations of sodium salicylate (SAL), the major metabolite of aspirin, can reduce CPS production by up to $70 \%[9,10]$. Aspirin also reduces the synthesis of prostaglandins (PGs) by inhibiting cyclooxygenase (COX)-mediated arachidonic acid metabolism [11], and therefore has attracted attention as a means of facilitating the killing of bacteria by leukocytes $[12,13]$. We hypothesized that SAL may enhance the 
phagocytosis of HV-KP by leukocytes as a result of reduced CPS production. In the present study, we tried to examine this hypothesis by investigating the effect of SAL on bacterial survival, hypermucoviscosity, CPS production, leukocyte phagocytosis, and bactericidal activity against HV-KP. Furthermore, we conducted a prospective study to evaluate the relationship between aspirin usage and KP-mediated invasive syndrome.

\section{Methods}

\section{Ethics statement}

All the protocols used in the present study have been approved by the Institutional Review Board at Kaohsiung Chang Gung Memorial Hospital (KCGMH; approval nos. 97-0599B and 100-0629B). Informed consent was not required in participants because of the observational nature of the study (97-0599B). In addition, 5 healthy male volunteers provided their written informed consents to participate in the study (100-0629B).

\section{Study design and participants}

In this prospective study, patients who were admitted to the KCGMH between January 1, 2008, and December 31, 2010, with community-acquired mono-microbial bacteraemia caused by KP were enrolled for the investigation of risk factors for KP-mediated invasive syndrome. Only the $\mathrm{KP}$ isolates from the participants' first blood collection were used in this study. Peripheral blood samples were also collected from 5 healthy male volunteers aged between 25 and 40 years old and used in the leukocyte phagocytosis and bactericidal activity assays as described below.

The diagnosis of KP-mediated invasive syndrome was made when the criteria for sepsis were met [14] plus the presence of at least one of the following infections: liver abscess, meningitis, empyema, mycotic aneurysm, necrotising fasciitis or endophthalmitis [15]. To investigate the risk factors of KP-mediated invasive syndrome, the following clinical variables were assessed: age, sex, comorbidities (including DM, liver cirrhosis, malignancy, chronic renal failure, and biliary tract disease), and a history of receiving proton-pump inhibitors or aspirin or antibiotics in the previous month prior to the collection of their first KP-positive blood culture. To monitor the glycemic control [16], the hemoglobin A1c $\left(\mathrm{HbA}_{1 \mathrm{c}}\right)$ was checked when diabetic patients were enrolled in this study. Controls were retrieved from a computer-aided selection of eligible individuals who community onset bacteremia caused by other than KP. Each subject was age-matched, and the ratio of case of KP invasive syndrome to control patients was 1:1.

\section{Reagents}

We purchased $10 \mathrm{mg} / \mathrm{mL} \mathrm{SAL}, 50 \mathrm{mM}$ ethylenediamine tetra-acetic acid, and $25 \mathrm{mM}$ ethylene-bis (oxyethylenenitrile) tetra-acetic acid from Sigma-Aldrich (St. Louis, MO). All stock solutions were prepared in deionized water, brought to $\mathrm{pH} 7.5$ using $10 \mathrm{~N} \mathrm{NaOH}$, and filter sterilized. Trypticase soy agar (TSA) and broth (TSB) media (Becton Dickinson, Franklin Lakes, NJ) were used for bacterial cultures. In some experiments, various concentrations of SAL were added into the growth media as indicated in the following experiments. The $\mathrm{pH}$ of the growth media with and without SAL was not significantly different.

\section{Bacterial isolates, hypermucoviscosity phenotype, and serotype determination}

All KP isolates were identified using standard methods. The HV phenotype was identified using a modified string test [17], with a viscous string longer than $10 \mathrm{~mm}$ as the criteria for a positive result. $K_{1}$ serotyping was assessed by polymerase chain reaction and capsular swelling technique [1]. A KP-M1 (serotype $K_{1}$ ) strain was isolated from an enrolled patient with invasive syndrome presenting as liver abscess and endophthalmitis and was used as the representing strain in the following experiments.

\section{Effects of SAL on live KP}

To exclude the possibility that SAL possesses direct bactericidal properties against KP, broth cultures of KP-M1 were incubated in the presence of various concentrations $(0,10,30$ and $300 \mu \mathrm{g} / \mathrm{mL})$ of SAL. After 2, 4, 8, 10, 12, and 24 hours, the number of surviving bacteria was monitored by reading the absorbance at $490 \mathrm{~nm}$. We further investigated the SAL effect on the hypermucoviscosity of KP. The KP-M1 colonies were cultured in plate media containing 0,10 , or $30 \mu \mathrm{g} / \mathrm{mL}$ of $\mathrm{SAL}$, respectively, and incubated overnight at $37^{\circ} \mathrm{C}$. The length of the mucoviscous string was measured [17].

\section{Quantification of CPS}

The CPS concentration was determined by a modified carbazole assay for uronic acid (UA) [18] after total CPS was quantitatively extracted from whole bacterial cultures [19]. A $0.5-\mathrm{mL}$ sample was mixed with $3 \mathrm{~mL}$ of $0.025 \mathrm{M}$ sodium tetraborate (VWR, Radnor, PA) in sulfuric acid, and heated at $100^{\circ} \mathrm{C}$ for $10 \mathrm{~min}$. After cooling, $0.1 \mathrm{~mL}$ of $0.125 \%$ carbazole (Sigma-Aldrich, St. Louis, MO) in absolute ethanol was added, and the samples were heated for another $15 \mathrm{~min}$. The absorbance of the sample at $530 \mathrm{~nm}$ was measured, and the concentration of UA was extrapolated from a standard curve that was constructed using glucuronolactone (Sigma-Aldrich, St. Louis, MO) standards. To quantify CPS after SAL treatment, bacterial colonies were cultured in media containing 0,10 , or $30 \mu \mathrm{g} / \mathrm{mL}$ SAL and incubated overnight at $37^{\circ} \mathrm{C}$. All samples were assayed at least 3 times. The quantities of CPS detected were expressed as micrograms of UA per $10^{11}$ colony forming units ( $\mu \mathrm{g}$ UA/ $10^{11} \mathrm{cfu}$ ). 
Flow cytometric analysis of leukocyte phagocytosis

The KP-M1 bacteria were cultured overnight at $37^{\circ} \mathrm{C}$ on TSA media containing 0,10 , or $30 \mu \mathrm{g} / \mathrm{mL}$ SAL. The bacterial colonies were irradiated with ultraviolet light then were sub-cultured in plate to confirm sterile after overnight incubation. The irradiated cultures were resuspended in carbonate buffer containing $0.1 \%$ fluorescein isothiocyanate (FITC). The FITC-stained KP (FITC-KP) cells were counted using a bacterial cytometer and a fluorescence microscope. The FITC-KP cells were analyzed using a FACS Calibur flow cytometer (BD Biosciences, San Jose, CA) to verify that bacterial staining was uniform throughout each sample.

Phagocytosis was measured using a standard assay [20]. Neutrophil suspensions and pooled human sera were collected from healthy human volunteers. The neutrophil suspension was adjusted to contain $1 \times 10^{6}$ cells in $100 \mu \mathrm{L}$ PBS and was then combined with $600 \mu \mathrm{L}$ PBS and $100 \mu \mathrm{L}$ pooled human serum (10\% v/v opsonization). Multiple volumes of $200 \mu \mathrm{L}$ of FITC-KP cells containing approximately $4 \times 10^{7} \mathrm{cfu} / \mathrm{mL}$ were added to $800 \mu \mathrm{L}$ of the neutrophilcontaining PBS to produce a final volume of $1.0 \mathrm{~mL}$ at a multiplicity of infection of 10:1. Each tube was incubated in a shaking water bath at $37^{\circ} \mathrm{C}$ for $60 \mathrm{~min}$. Ethidium bromide was added to suppress extracellular fluorescence. A FACScan instrument (BD Biosciences, San Jose, CA) was used to detect FITC fluorescence at $488 \mathrm{~nm}$, and $2 \times 10^{4}$ cells were processed using the Cellquest version 1.0 software (BD Biosciences, San Jose, CA). By assessing phagocytosis of unstained and FITC-stained bacteria, the boundary of positive and negative fluorescence was established, and the percentage of ingested bacteria was determined.

\section{Ex vivo human leukocyte bactericidal activity assay}

Bactericidal activity was measured using a standard assay method [21]. The KP-M1 organisms were cultured on TSA media containing 0 or $30 \mu \mathrm{g} / \mathrm{mL}$ SAL, respectively, and incubated overnight at $37^{\circ} \mathrm{C}$. The KP-M1 organisms were opsonized by the addition of $10 \%$ pooled human serum collected from 5 healthy male volunteers who did not take any aspirin prior to the donation. The suspension was mixed at a multiplicity of infection of 10:1 to whole blood leukocytes, which had been collected from volunteers either before or one hour after a 100-mg oral dose of aspirin. Samples were collected one hour later, and diluted in $\mathrm{H}_{2} \mathrm{O}(\mathrm{pH} 11.0$, adjusted by $\mathrm{NaOH}$ ) to lyse the leukocytes and disperse the bacteria for the powerplate colony assay [22]. All tests were performed in triplicate to ensure reproducibility.

\section{Statistical analysis}

The categorical variables were compared using the chi-square test or the Fisher exact test, as appropriate. A multivariate logistic regression model was used to evaluate risk factors for invasive syndrome by calculating the odds ratio (OR) and 95\% confidence interval (CI) of each clinical variable. All experimental data were expressed as the mean \pm standard deviation. Differences among the results of the string test $(\mathrm{mm})$ and phagocytosis (gate \%) were analyzed using the Mann-Whitney U-test. The counts of surviving bacteria were analyzed using the log rank test. All statistical analyses were twosided, and a $p$ value less than 0.05 was considered to indicate a statistically significant result.

\section{Results}

\section{Risk factors for KP-mediated invasive syndrome}

Of the 408 patients with community-acquired mono-KP bacteremia, 76 (18.6\%) had invasive syndrome. Based on results of the modified string test, the $\mathrm{HV}$ phenotype was identified in 147 (36.0\%) isolates. As demonstrated in Table 1, invasive syndrome occurred more often in HV-KP-infected patients $(p<0.01)$. A large proportion $(75,42.9 \%)$ of the patients studied also suffered from DM. The proportion of KP infection-related invasive syndrome was significantly higher among these DM patients $(p=0.04)$, especially those with poor glycemic control $\left(\mathrm{HbA}_{1 \mathrm{c}} \geq 9 \% ; p<0.01\right)$. Aspirin therapy received during the month prior to the diagnosis of the KP infection was found in 58 (14.2\%) patients, and they were less likely to develop invasive syndrome $(p<0.01$; Table 1$)$. Further multivariate analysis indicated that communityacquired KP-bacteremic patients who were infected by strains expressing the HV phenotype (odds ratio [OR], 31.07; 95\% confidence interval [CI], 13.55-71.22; $p<$ 0.01 ) and DM patients with poor glycemic control (OR, 2.46; 95\% CI, 1.27-4.77; $p<0.01$ ) were at increased risk, whereas those who had a recent use of aspirin (OR, 0.17; 95\% CI, 0.04-0.79; $p=0.02$ ) were at a lower risk of acquiring $\mathrm{KP}$-associated invasive syndrome. Comparing with age-matched control group (Table 1), it revealed that patients with KP-associated invasive syndrome were more likely to have DM $(p=0.01)$, DM with poor glycemic control $(p=0.02)$ and less likely to have malignancy $(p<0.01)$.

\section{Effects of SAL on live KP}

No significant difference was found between the number of viable KP-M1 cells cultured with or without the presence of various concentrations of SAL. However, compared to those grown in other concentrations of SAL, viable KP-M1 cells were much less in $300 \mu \mathrm{g} / \mathrm{mL}$ of SAL (Figure 1). It suggests that at high concentration of SAL $(300 \mu \mathrm{g} / \mathrm{mL})$ might interfere with some cellular processes or bind to essential elements, which results in reduction in growth. 
Table 1 Comparisons of differences between patients with community-acquired $K$. pneumoniae bacteremia and control group

\begin{tabular}{|c|c|c|c|c|c|}
\hline \multirow{3}{*}{ Variable } & \multirow{2}{*}{\multicolumn{2}{|c|}{$\frac{\text { Community-acquired } K . \text { pneumoniae bacteremia }}{\text { Invasive syndrome }(\%)}$}} & \multirow[b]{3}{*}{$p$ value } & \multirow[b]{3}{*}{ Control $(\%)(n=76)$} & \multirow[b]{3}{*}{$p$ value } \\
\hline & & & & & \\
\hline & Yes $(n=76)$ & No $(n=332)$ & & & \\
\hline Hypermucoviscosity phenotype of $K$. pneumoniae & $69(90.8)$ & $78(23.5)$ & $<0.01^{*}$ & & \\
\hline Male & $40(52.6)$ & $150(45.2)$ & 0.25 & $40(52.6)$ & 0.99 \\
\hline Age $\geq 60$ yrs & $39(51.3)$ & $128(38.6)$ & 0.05 & $39(51.3)$ & 0.99 \\
\hline Diabetes mellitus (DM) & $41(53.9)$ & $134(40.4)$ & 0.04 & $25(32.9)$ & 0.01 \\
\hline DM with poor glycemic control $\left(\mathrm{HbA}_{1 c} \geq 9 \%\right)$ & $32(42.1)$ & $75(22.6)$ & $<0.01^{*}$ & $17(22.4)$ & 0.02 \\
\hline Cardiovascular diseases & $17(22.4)$ & 65 (19.6) & 0.69 & $21(27.6)$ & 0.57 \\
\hline Liver cirrhosis & $5(6.6)$ & 45 (13.6) & 0.12 & $10(13.2)$ & 0.28 \\
\hline Chronic renal failure & $10(13.2)$ & $35(10.5)$ & 0.54 & $18(23.7)$ & 0.14 \\
\hline Malignancy & $4(5.3)$ & $33(9.9)$ & 0.27 & $18(23.7)$ & $<0.01$ \\
\hline Biliary tract diseases & $2(2.6)$ & $20(6.0)$ & 0.39 & $8(10.5)$ & 0.10 \\
\hline Absence of underlying diseases & $12(15.8)$ & $44(13.3)$ & 0.69 & $9(11.8)$ & 0.64 \\
\hline \multicolumn{6}{|l|}{ Therapy in the month prior to the infection } \\
\hline Proton-pump inhibitors & $8(10.5)$ & $24(7.2)$ & 0.47 & $15(19.7)$ & 0.17 \\
\hline Aspirin & $2(2.6)$ & $56(16.9)$ & $<0.01^{*}$ & $8(10.5)$ & 0.10 \\
\hline Antibiotics & $5(6.6)$ & $28(8.4)$ & 0.76 & $12(15.8)$ & 0.12 \\
\hline
\end{tabular}

${ }^{+}$Age-matched analysis (K. pneumoniae invasive syndrome vs. control group).

*Results of multivariate analysis indicated that community-acquired $K$. pneumoniae bacteremic patients who were infected by strains expressing the hypermucoviscosity phenotype (odds ratio [OR], 31.07; $95 \%$ confidence interval [Cl], 13.55-71.22; $p<0.01$ ) and diabetic patients with poor glycemic control (OR, 2.46; $95 \% \mathrm{Cl}$, $1.27-4.77 ; p<0.01)$ were at increased risk, whereas those who had recent therapy with aspirin $(\mathrm{OR}, 0.17 ; 95 \% \mathrm{Cl}, 0.04-0.79 ; p=0.02)$ were at lower risk of acquiring K. pneumoniae-associated invasive syndrome.

Effect of SAL on hypermucoviscosity and CPS production Incubation of KP-M1 colonies on TSB agar media containing 10 or $30 \mu \mathrm{g} / \mathrm{mL}$ SAL significantly decreased the incidence of the $\mathrm{HV}$ phenotype, according to the results of the modified string test $(p<0.01$, Figure $2 \mathrm{~A}$ and $2 \mathrm{~B})$. The quantity of CPS found in KP-M1 cells was equivalent to

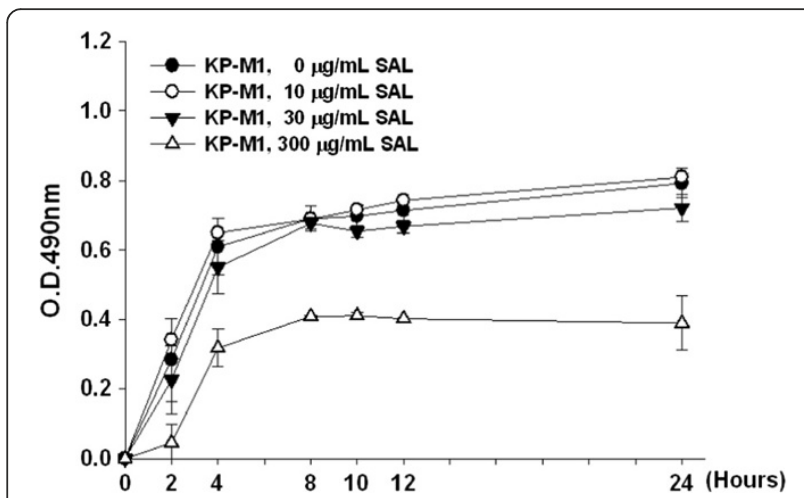

Figure 1 Effects of sodium salicylate (SAL) on live KP-M1 strain of Klebsiella pneumoniae. After 24-h incubation with various concentrations of SAL, the growth of KP-M1 cells were monitored by reading the absorbance (OD) at $490 \mathrm{~nm}$. The growth of KP-M1 cells cultured in media containing 10 or $30 \mu \mathrm{g} / \mathrm{mL}$ SAL did not differ from those cultured without SAL. However, the KP-M1 cells decreased in growth when the bacteria were cultured in the presence of $300 \mu \mathrm{g} / \mathrm{mL}$ SAL.
$2475.87 \pm 175.09 \mu \mathrm{g} \mathrm{UA} / 10^{11} \mathrm{cfu}$. When the KP-M1 bacteria were cultured in 10 or $30 \mu \mathrm{g} / \mathrm{mL} \mathrm{SAL}$, the quantity of CPS decreased to $1821.27 \pm 73.76 \mu \mathrm{g} \mathrm{UA} / 10^{11} \mathrm{cfu}$ and $1574.48 \pm 86.90 \mu \mathrm{g} \mathrm{UA} / 10^{11} \mathrm{cfu}$, respectively. This equaled to $26.4 \%(p<0.01)$ and $34.4 \%(p<0.01)$ reduction of CPS levels, respectively, as compared with the mean control values. Therefore, the quantity of CPS could be significantly reduced in the presence of SAL.

\section{Effects of SAL on leukocyte phagocytosis and ex vivo leukocyte bactericidal activity}

The difference between the rate of KP-M1 phagocytosis by neutrophils before and after co-incubation with 10 or $30 \mu \mathrm{g} / \mathrm{mL}$ SAL was significant $(p<0.01$; Figure $3 \mathrm{~A}$ and $3 B)$. The KP-M1 was significantly more susceptible to phagocytosis after incubation with 10 or $30 \mu \mathrm{g} / \mathrm{mL}$ SAL. The ex vivo bactericidal activity of leukocytes from healthy volunteers that were collected one hour following the oral administration of $100 \mathrm{mg}$ of aspirin was significantly greater than that of leukocytes collected from the same volunteers before the aspirin treatment $(p<0.01$; Figure 4$)$. The results indicated that aspirin was able to enhance significantly the whole blood leukocyte bacterial killing despite the pre-incubation of KP-M1 with SAL. 

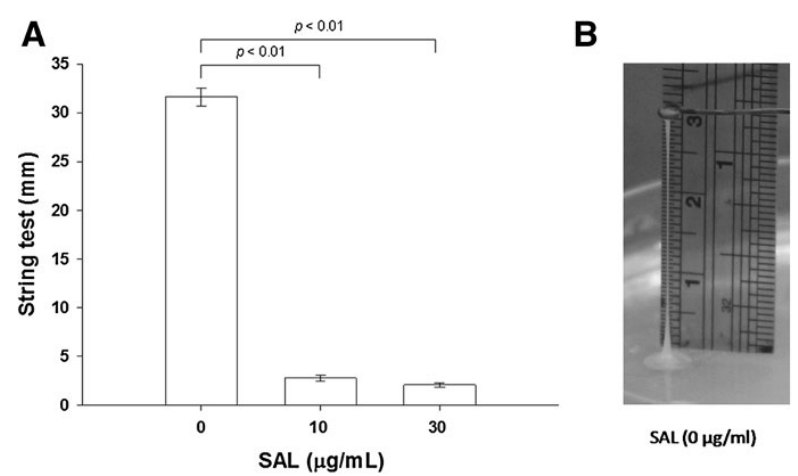

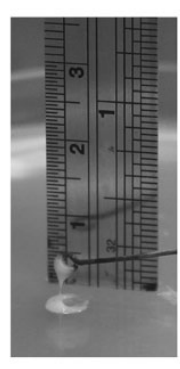

$\mathrm{SAL}(10 \mu \mathrm{g} / \mathrm{ml})$

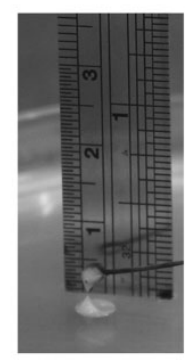

$\mathrm{SAL}(30 \mu \mathrm{g} / \mathrm{ml})$

Figure 2 String test for hypermucoviscosity (HV) in the KP-M1 strain of Klebsiella pneumoniae. (A) Each closed bar indicates the mean \pm standard deviation in millimeters for the results of string tests among KP-M1 organisms treated with various concentrations of sodium salicylate (SAL) as indicated. The asterisk indicates the significant changes among the results with different SAL concentrations $(p<0.01)$. (B) Representative photographs of the stretched, mucoviscous string from single colonies of KP-M1 organisms treated with various concentrations of SAL as indicated.

\section{Discussion}

The CPS surrounding KP bacteria enables the bacteria to evade phagocytosis $[23,24]$. Reduction of the CPS by SAL may therefore attenuate the pathogenicity of KP strains. Cell death could not explain the decrease in CPS observed in our experiments because viable bacterial colonies remained unchanged in the presence of various levels of SAL that were optimized for reducing the CPS. These levels of SAL are within the therapeutic range measured in serum after ingestion of low dose aspirin [25]. Hence, with the common oral use of aspirin, the effect of CPS reduction in KP could occur.

Epidemiological studies have demonstrated that the majority of KP infections are preceded by the colonization of the gastrointestinal tract [26]. Patients infected with HVKP strains and DM patients with uncontrolled glycemia are known to be at increased risks for invasive syndrome $[3,17]$. Our results indicated that patients who had used aspirin during the month prior to the diagnosis of community-acquired KP bacteremia appeared to be associated with a lower risk for invasive syndrome.
The mechanism by which CPS is reduced in the presence of SAL remains unclear. Metal ions are cofactors for some of the enzymes involved in polysaccharide synthesis [27]. SAL has been identified as the biosynthetic precursor of the aromatic ring in pyochelin, an ironchelating siderophore [28]. Divalent cations also play an important role in the stabilization of bacterial outermembrane proteins [28]. The reduction of CPS production by SAL is likely the result of either the modulation of enzyme activity in CPS synthesis pathways or the disruption of the functional integrity of KP outer membranes.

Neutrophils have been shown to be capable of ingesting and killing encapsulated KP in the presence of complement [23]. SAL-mediated capsular suppression was shown to enable serum complement, such as $\mathrm{C} 3$, to bind to sites on the bacteria that were otherwise masked by the capsule [10]. In our experiments, homogenization with SAL removed $20 \%$ to $40 \%$ of the CPS production and resulted in approximately $40 \%$ to $60 \%$ increase in the rate of phagocytosis. Although homogenization with low doses of aspirin did not completely remove the
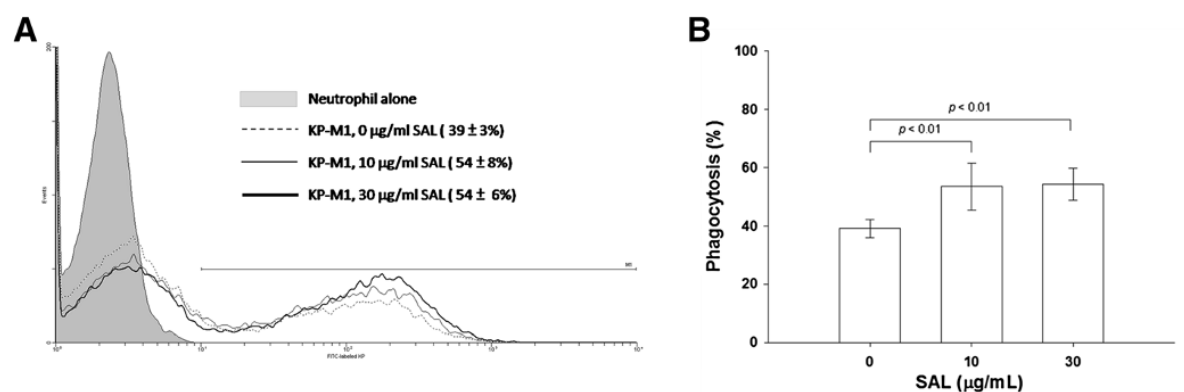

Figure 3 Flow cytometry assessment of neutrophil phagocytosis. (A) Trypan blue quenching of the extracellular fluorescence of bound bacteria and phagocytosed bacteria by neutrophils after incubation at $37^{\circ} \mathrm{C}$ for $60 \mathrm{~min}$ were evaluated by flow cytometry. (B) The KP-M1 bacteria were significantly more susceptible to phagocytosis by neutrophils after incubation with 10 or $30 \mathrm{\mu g} / \mathrm{mL}$ sodium salicylate (SAL). 


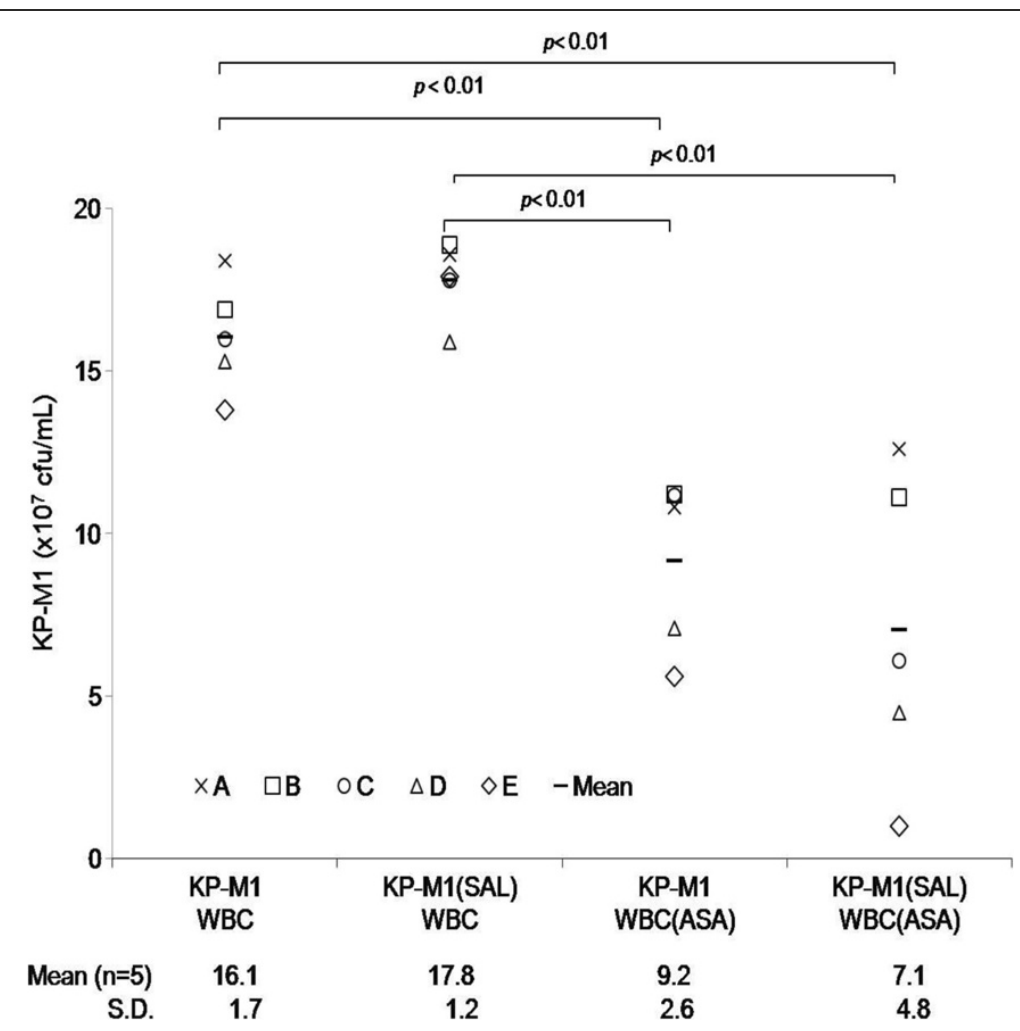

Figure 4 Ex vivo human leukocyte bactericidal activity assay. Human peripheral white blood cells (WBCs) from 5 healthy males were collected before and one hour after oral administration of $100 \mathrm{mg}$ of aspirin (ASA), and incubated, respectively, with the KP-M1 strain of Klebsiella pneumoniae (KP) which had been previously opsonized with normal human serum. The ASA treatment significantly enhanced the leukocyte killing of KP-M1 $(p<0.01)$, disregard whether or not the KP-M1 organisms were pre-incubated with sodium salicylate $(S A L, 30 \mu \mathrm{g} / \mathrm{mL})$.

capsule, it may render the bacteria to become more susceptible to phagocytosis by neutrophils. The loss of capsular materials may help to expose the cell surface of KP to the host defense mechanisms and thus shorten the time required for the clearance of the infection [29].

Recently, aspirin was shown to promote the killing of S. pneumoniae by blood leukocytes through COX inhibition [13]. Similarly, in the present study, we further demonstrated that aspirin enhanced the killing of HVKP by leukocytes. However, the survival rates of KP-M1 cells did not have significant changes whether or not the bacteria were pretreated with SAL. KP organisms have been shown to remain alive after being phagocytosed [6]. Therefore, SAL treatment may only increase the uptake of the bacteria by neutrophils without affecting the extent of neutrophil-mediated intracellular killing. It is until the addition of aspirin-pretreated leukocytes that an enhance leukocyte bactericidal effect is observed.

Although the multivariate analysis showed significant protection in patients who were treated with aspirin, their blood levels of SAL were not documented. In addition, we did not perform stool sampling for KP isolation, and so the contribution of aspirin to patients' carriage could not be assessed. It was an uncontrolled observational study. The patients who suffered from KP bacteremia may be severe and referred from other hospitals, the reliability of medication history may be a confounding factor. Therefore, we could not exclude the possibilities of other unmeasured potential confounders that may affect the association of aspirin with the enhanced leukocyte killing, thus limiting our ability to delineate the true relationship between aspirin and invasive HV-KP infection. Lastly, only one strain (KPM1; serotype $K_{1}$ ) was assessed in this study. However, epidemiological reports have indicated that capsular serotype $K_{1}$ is the most prevalent in primary KP liver abscess $[2,7]$. Serotype $K_{1}$ strains were also significantly more virulent than non- $\mathrm{K}_{1}$ strains, in terms of higher in vitro serum resistance and greater risk of septic ocular or central nervous system complications for primary liver abscess. ${ }^{7}$ Therefore, our results derived from the representative serotype $K_{1}$ strain might be extrapolated for the majority of KP strains that caused invasive syndrome.

\section{Conclusions}

In summary, our data indicated that SAL could affect the CPS production and the HV phenotype in KP. It also 
promotes the leukocyte phagocytosis and bactericidal activity against KP. The in vitro and ex vivo effects of SAL on serotype $K_{1} \mathrm{KP}$ warrant further investigation on the role of aspirin as an adjunct therapy for KP-mediated invasive syndrome.

\section{Abbreviations}

Cl: Confidence interval; COX: Cyclooxygenase; CPS: Capsular polysaccharide; DM: Diabetic mellitus; FITC: Fluorescein isothiocyanate;

HV: Hypermucoviscosity; KP: Klebsiella pneumoniae; OR: Odds ratio;

PG: Prostaglandin; SAL: Sodium salicylate; UA: Uronic acid.

\section{Competing interests}

The authors declare that they have no competing interests.

\section{Authors' contributions}

$\mathrm{CHL}$, LHS, JWL, CCC, and KDY participated in the design of this study. CHL, $\mathrm{CCC}$, and RFC developed the in vitro assay. CHL, LHS, and KDY wrote the manuscript. All authors read and approved the final manuscript.

\section{Acknowledgements}

This study is supported by grants from the National Science Council, Taiwan (NSC 100-2314-B-182A-026) and Chang Gung Memorial Hospital, Taiwan (CMRPG 8A0311). None of the author has potential conflicts of interest.

\section{Author details}

'Division of Infectious Diseases, Kaohsiung Chang Gung Memorial Hospital, Kaohsiung, Taiwan. ${ }^{2}$ Chang Gung University College of Medicine, Kaohsiung, Taiwan. ${ }^{3}$ Department of Laboratory Medicine, Chang Gung Memorial Hospital, Linkou, Taiwan. ${ }^{4}$ Chang Gung University, College of Medicine, Kweishan, Taoyuan, Taiwan. ${ }^{5}$ Department of Medical Research, Show Chwan Memorial Hospital, 6 Lukong Road, Lugang, Changhua 505, Taiwan.

Received: 23 October 2013 Accepted: 23 January 2014

Published: 30 January 2014

\section{References}

1. Wang JH, Liu YC, Lee SS, Yen MY, Chen YS, Wann SR, Lin HH: Primary liver abscess due to Klebsiella pneumoniae in Taiwan. Clin Infect Dis 1998, 26:1434-1438

2. Ko WC, Paterson DL, Sagnimeni AJ, Hansen DS, Von Gottberg A, Mohapatra S, Casellas JM, Goossens H, Mulazimoglu L, Trenholme G, Klugman KP, McCormack JG, Yu VL: Community-acquired Klebsiella pneumoniae bacteremia: global differences in clinical patterns. Emerg Infect Dis 2002, 8:160-166.

3. Lee CH, Liu JW, Su LH, Chien CC, Li CC, Yang KD: Hypermucoviscosity associated with Klebsiella pneumoniae-mediated invasive syndrome: a prospective cross-sectional study in Taiwan. Int J Infect Dis 2010, 14:e688-e692

4. Lin JC, Chang FY, Fung CP, Xu JZ, Cheng HP, Wang JJ, Huang LY, Siu LK: High prevalence of phagocytic-resistant capsular serotypes of Klebsiella pneumoniae in liver abscess. Microbes Infect 2004, 6:1191-1198.

5. Lin JC, Siu LK, Fung CP, Tsou HH, Wang JJ, Chen CT, Wang SC, Chang FY: Impaired phagocytosis of capsular serotype K1 or K2 Klebsiella pneumoniae in type 2 diabetic mellitus patients with poor glycemic control. J Clin Endocrinol Metab 2006, 91:3084-3087.

6. Lin JC, Chang FY, Fung CP, Yeh KM, Chen $C$, Tasi YK, Siu LK: Do neutrophils play a role in establishing liver abscesses and distant metastases caused by Klebsiella pneumoniae. PLoS One 2010, 5:e15005.

7. Fang CT, Lai SY, Yi WC, Hsueh PR, Liu KL, Chang SC: Klebsiella pneumoniae genotype K1: an emerging pathogen that causes septic ocular or central nervous system complications from pyogenic liver abscess. Clin Infect Dis 2007, 45:284-293.

8. Pollack M: Significance of circulating capsular antigen in Klebsiella infections. Infect Immun 1976, 13:1543-1548.

9. Domenico P, Schwartz S, Cunha BA: Reduction of capsular polysaccharide production in Klebsiella pneumoniae by sodium salicylate. Infect Immun 1989, 57:3778-3782
10. Domenico P, Salo RJ, Straus DC, Hutson JC, Cunha BA: Salicylate or bismuth salts enhance opsonophagocytosis of Klebsiella pneumoniae. Infection 1992, 20:66-72.

11. Vane JR: Inhibition of prostaglandin synthesis as a mechanism of action for aspirin-like drugs. Nat New Biol 1971, 231:232-235.

12. Aronoff DM, Lewis C, Serezani CH, Eaton KA, Goel D, Phipps JC, Peters-Golden M, Mancuso P: E-prostanoid 3 receptor deletion improves pulmonary host defense and protects mice from death in severe Streptococcus pneumoniae infection. J Immunol 2009, 183:2642-2649.

13. Stables MJ, Newson J, Ayoub SS, Brown J, Hyams CJ, Gilroy DW: Priming innate immune responses to infection by cyclooxygenase inhibition kills antibiotic-susceptible and -resistant bacteria. Blood 2010, 116:2950-2959.

14. American College of Chest Physicians/Society of Critical Care Medicine Consensus Conference: Definitions for sepsis and organ failure and guidelines for the use of innovative therapies in sepsis. Crit Care Med 1992, 20:864-874.

15. Yu VL, Hansen DS, Ko WC, Sagnimeni A, Klugman KP, von Gottberg A, Goossens H, Wagener MM, Benedi VJ, International Klebseilla Study Group: Virulence characteristics of Klebsiella and clinical manifestations of $K$. pneumoniae bloodstream infections. Emerg Infect Dis 2007, 13:986-993.

16. American Diabetes Association: Standards of medical care in diabetes-2009. Diabetes Care 2009, 32:513-561.

17. Lee HC, Chuang YC, Yu WL, Lee NY, Chang CM, Ko NY, Wang LR, Ko WC Clinical implications of hypermucoviscosity phenotype in Klebsiella pneumoniae isolates: association with invasive syndrome in patients with community-acquired bacteremia. J Intern Med 2006, 259:606-614.

18. Bitter T, Muir HM: A modified uronic acid carbazole reaction. Anal Biochem 1962, 4:330-334

19. Domenico P, Diedrich DL, Cunha BA: Quantitative extraction and purification of exopolysaccharides from Klebsiella pneumoniae. J Microbiol Methods 1989, 9:211-219.

20. Heinzelmann M, Gardner SA, Mercer-Jones M, Roll AJ, Polk HC Jr: Quantification of phagocytosis in human neutrophils by flow cytometry. Microbiol Immunol 1999, 43:505-512.

21. Hampton MB, Vissers MC, Winterbourn CC: A single assay for measuring the rates of phagocytosis and bacterial killing by neutrophils. J Leukoc Biol 1994, 55:147-152.

22. Green JN, Winterbourn CC, Hampton MB: Analysis of neutrophil bactericidal activity. Methods Mol Biol 2007, 412:319-332.

23. Williams $P$, Lambert PA, Haigh CG, Brown MR: The influence of the $O$ and $\mathrm{K}$ antigens of Klebsiella aerogenes on surface hydrophobicity and susceptibility to phagocytosis and antimicrobial agents. J Med Microbiol 1986, 21:125-132.

24. Domenico P, Diedrich DL, Straus DC: Extracellular polysaccharide production by Klebsiella pneumoniae and its relationship to virulence. Can J Microbiol 1985, 31:472-478.

25. Cerletti C, Dell'Elba G, Manarini S, Pecce R, Di Castelnuovo A, Scorpiglione N, Feliziani V, de Gaetano G: Pharmacokinetic and pharmacodynamic differences between two low dosages of aspirin may affect therapeutic outcomes. Clin Pharmacokinet 2003, 42:1059-1070.

26. Fung $C P$, Lin $Y T$, Lin JC, Chen TL, Yeh KM, Chang FY, Chuang HC, Wu HS, Tseng CP, Siu LK: Klebsiella pneumoniae in gastrointestinal tract and pyogenic liver abscess. Emerg Infect Dis 2012, 18:1322-1325.

27. Sutherland IW: Bacterial polysaccharides-their nature and production. In Surface carbohydrates of the prokaryotic cell. Edited by Sutherland IW. New York: Academic Press; 1977:27-96.

28. Leive $L$ : Studies on the permeability change produced in coliform bacteria by ethylenediamine tetraacetate. J Biol Chem 1968, 243:2373-2380.

29. Salo RJ, Domenico P, Tomás JM, Straus DC, Merino S, Benedí VJ, Cunha BA: Salicylate-enhanced exposure of Klebsiella pneumoniae subcapsular components. Infection 1985, 23:371-377.

doi:10.1186/1471-2334-14-47

Cite this article as: Lee et al:: Aspirin enhances opsonophagocytosis and is associated to a lower risk for Klebsiella pneumoniae invasive syndrome. BMC Infectious Diseases 2014 14:47. 